\title{
Psychiatrists' Association Of Nepal - Future Of Psychiatric Services In Nepal - A Mission And Vision Document
}

\section{Koirala NR}

President, Psychiatrists' Association of Nepal; Professor and Head, Department of Psychiatry and Mental Health, NMC, Biratnagar, Nepal

\section{MISSION:}

- The Psychiatrists' Association of Nepal (PAN) is a non-profit professional organization of Nepalese psychiatrists, established in the year 1990, committed to the welfare of its members and to the advancement of mental health care, education, advocacy and research in the country.

- The Psychiatrists' Association of Nepal envisions mental health services in the country that fosters a future when everyone with a mental illness will recover, a future when mental illness can be prevented, treated or cured, a future when mental illnesses are detected early, and a future when everyone with a mental illness at any stage of life has access to effective treatments and supports-essential for living, working, learning and participating fully in the community.

- The Psychiatrists' Association of Nepal envisions a system of care that is adequately funded, consumer and family driven, and geographically accessible. This system should continue to linked with community-based mental health services, should include all available inpatient and out-patient services, and will be guided by evidence based research and practices.

- The Psychiatrists' Association of Nepal envisions that the PAN will be recognized as a premier organization of Psychiatrists in Nepal and South Asian Region by the year 2020 .

\section{GOALS:}

1. Advancement in standards of Mental Health Services in the country:

- Psychiatrists' Association of Nepal will take a leadership role in the development and implementation of a Mental Health Policy and mental health Act.

- Increased accessibility of psychiatrists and other mental health care services in underserved and poorly served population/areas in the country through expansion of community mental health services in collaboration with governmental (PHC, District Hospitals, Universities training programs-eg. One month Community Posting of Undergraduate students in TU etc) and Non-governmental Organizations,

- Increased awareness of the rights and responsibilities of patients and their families.

To achieve all above-mentioned mission, vision and goals the present executive committee of PAN will put all its efforts in the implementation of National Mental Health Policy and National Mental Health Act.

The salient features of Nepal's Mental Health Policy:

- The National Mental Health Policy of Government of Nepal was formulated in the year 1996 and in the same year it has endorsed by the Parliament and adopted/incorporated in to $9^{\text {th }}$ Five Year National Plan by the Government of 
Nepal. Key components of the policy include: (1) to ensure the availability and accessibility of minimum mental health services for all the population of Nepal; (2) to prepare human resources in the area of mental health; (3) to protect the fundamental human rights of the mentally ill; and (4) to improve awareness about mental health. There is an essential drug list for different levels of health institutions.

The salient features of Nepal's Mental Health Legislation/Act

- There is no separate Mental Health Legislation as yet, but a final draft of mental health legislation has been prepared in the year of 2006 by a team of expert mental health professionals, $\mathrm{WHO}$ representative, Human right activist, representative of national Planning Commission and Government of Nepal and other stakeholders (NGO, INGOs). It has already been submitted to the Ministry of Health for review and finalization in the year 2010. We hope that it will be endorsed by the Parliament and implemented by the Government of Nepal soon.

\section{Advancement in standards of Mental Health Education:}

- The Undergraduate Curricula for Psychiatry should comply with the Nepal Medical Council Core Curricula for undergraduate Psychiatry and should be uniform throughout the country.

Present scenario:

Credit Hours:

TU-Theory 45 hours and Clinical Postings-162 Hours,

KU-Theory 25 Hours, Clinical Posting- 60 Hours

BPKIHS: Theory-55 Hrs, Clinical Postings-72 Hrs)
Evaluation process:

In TU and BPKIHS-Separate examination is mandatory as subsidiary subject of Internal Medicine-fullmarks-16 theory and 20 in practical) In KU-no separate examination, included in Internal Medicine)

- All Training Programs should comply with the Nepal Medical Council and World Federation of Medical Education Standards for Postgraduate Medical Education.

\section{Present scenario:}

Post-Graduate training programs TU, KU, NAMS and BPKIHS-all are different, no uniform curriculum and process of evaluation.

- The Curriculum for psychiatry should emphasize social, community, Child and adolescent and women mental health issues adequately.

- All members of PAN Should update their knowledge and competencies by attending regular continuing medical education activities hosted by the PAN and/or its international affiliated organizations.

- Psychiatrists' association of Nepal has already proposed to the Ministry of Health and Respective Universities in the country for the beginning of Superspeciality training programs in Child and adolescent Psychiatry, Addiction Psychiatry and Geriatric Psychiatry by the year 2015.

\section{Advancement in Mental Health Advocacy, Public Information and Mental Health Care:}

- The Psychiatrists' Association of Nepal will lead a leadership position in the advocacy for the promotion of mental health and welfare of persons with mental health conditions. 


\section{Advancement in Standard of Mental Health} Care:

- The standard of care will be based on accepted Clinical Practice Guidelines developed by the Task Force group of PAN and/or by internationally recognized professional organizations (WPA, WHO etc.)

- More Mental Health professionals including psychiatrists, Psychiatric nurses, Psychiatric social workers and Occupational Therapists will be trained in the field of mental health.

(Number of Psychiatrist $-64+8$ expatriates

Psychiatric Nurses- M.N. and MSc. 8, B.N-16.

No Psychiatric Social workers and Occupational Therapist yet in the country)

- The PAN will advocate for regular posting of Psychiatrists in district, zonal and Regional hospitals in the country, as guided by National Mental Health Policy.

- The total number of existing out-patient and in-patient services will be expanded by effective delivery of mental health services through Primary Health Care in the country. The existing in-patient beds will also be increased by double by the end of 2015.

\section{Advancement in Mental Health Research:}

- The research fund of the organization will be strengthened.

- The PAN will put its all efforts to establish and strengthen collaborative linkages and network programs with national and international research institutes.

- Basic studies in Psychiatry will be conducted and published, i.e., epidemiological studies, intervention research that provide a basis for management information system, relevant for mental health delivery system.

- More psychiatrists will be trained in the field of mental health research.

- The Journal of Psychiatrists' Association of Nepal (JPAN) will be published regularly. Its publication will be made quarterly a year instead of biannually and will be indexed.

- Seminars, Symposia, workshop, conferences and CME will be organised regularly at regional, national and international level.

\section{Promotion of WELFARE of Members:}

- The PAN will create a sustainable welfare fund to support its membership privileges (Near about NRS 14 lacks has already been collected and this amount will be increased by double at the end of 2015.

- All Members should adhere to the highest standards of ethical practice as guided by the PAN Code of Ethics.

- Medico-legal support related to the practice of Psychiatry will be available to its members if requires.

- A mechanism of Social Support will be available to its members in case of Death, Sickness, accidents and adverse life experiences, and their professional development (Training, Fellowships programs etc). 